\title{
The Expected Redshift Distribution of Gamma\#Ray Bursts
}

\section{Citation}

Bromm, Volker, and Abraham Loeb. 2002. "The Expected Redshift Distribution of Gamma\#Ray Bursts." The Astrophysical Journal 575 (1): 111-16. https://doi.org/10.1086/341189.

\section{Permanent link}

http://nrs.harvard.edu/urn-3:HUL.InstRepos:41393331

\section{Terms of Use}

This article was downloaded from Harvard University's DASH repository, and is made available under the terms and conditions applicable to Other Posted Material, as set forth at http:// nrs.harvard.edu/urn-3:HUL.InstRepos:dash.current.terms-of-use\#LAA

\section{Share Your Story}

The Harvard community has made this article openly available.

Please share how this access benefits you. Submit a story.

\section{Accessibility}




\title{
THE EXPECTED REDSHIFT DISTRIBUTION OF GAMMA-RAY BURSTS
}

\author{
Volker Bromm and ABraham Loeb \\ Astronomy Department, Harvard University, 60 Garden Street, Cambridge, MA 02138; \\ vbromm@cfa.harvard.edu, aloeb@cfa.harvard.edu \\ Received 2002 January 24; accepted 2002 April 12
}

\begin{abstract}
We predict the redshift distribution of gamma-ray bursts (GRBs), assuming that they trace the cosmic star formation history. We find that a fraction $\gtrsim 50 \%$ of all GRBs on the sky originate at a redshift $z \gtrsim 5$, even though the fraction of the total stellar mass formed by $z \sim 5$ is only $\sim 15 \%$. These two fractions are significantly different, because they involve different cosmological factors when integrating the star formation rate over redshift. Hence, deep observations of transient events, such as GRB afterglows or supernovae, provide an ideal strategy for probing the high-redshift universe. We caution, however, that existing or planned fluxlimited instruments are likely to detect somewhat smaller fractions of high-redshift bursts. For example, we estimate that the fraction of all bursts with redshifts $z \gtrsim 5$ is $\sim 10 \%$ in the case of the BATSE instrument and $\sim 25 \%$ in the case of Swift. We also show that the intrinsic distribution of GRB durations is bimodal but significantly narrower and shifted toward shorter durations than the observed distribution.
\end{abstract}

Subject headings: cosmology: theory — early universe — gamma rays: bursts

\section{INTRODUCTION}

Gamma-ray bursts (GRBs) are the brightest electromagnetic explosions in the universe (for a recent review, see Piran 2000). Popular models for their central engine divide into two main classes: (1) the collapse of a massive star to a black hole (BH; MacFadyen, Woosley, \& Heger 2001 and references therein); and (2) the coalescence of a binary system involving a neutron star (NS) and a $\mathrm{BH}$ or an NS as a companion (see, e.g., Eichler et al. 1989; Janka et al. 1999). The observed association of long-duration GRBs with starforming regions (Djorgovski et al. 2001c and references therein) and the possible supernova signatures in rapidly decaying afterglows (Bloom et al. 1999; Kulkarni et al. 2000; Reichart 2001) favor the first class. Both classes of models associate GRB progenitors with compact objects (BHs or NSs) that are the end products in the evolution of massive stars. Hence, the GRB formation history is expected to follow the cosmic star formation history (Totani 1997, 1999; Wijers et al. 1998; Blain \& Natarajan 2000) up to the highest redshifts $(z \sim 20)$, at which the first generation of stars may have formed (Barkana \& Loeb 2001). GRBs might therefore provide an ideal probe of cosmic star formation at all redshifts that, in particular, is unaffected by dust obscuration (see, e.g., Blain \& Natarajan 2000; Porciani \& Madau 2001). In fact, the top-heavy initial mass function (IMF) predicted for the first stars (Bromm, Coppi, \& Larson 1999, 2002; Abel, Bryan, \& Norman 2000, 2002; Nakamura \& Umemura 2001) favors massive stars that are the likely source of GRB progenitors.

GRB afterglows provide a unique probe of the high-redshift universe (Lamb \& Reichart 2000; Ciardi \& Loeb 2000). The bright, early, optical-UV luminosity of a GRB afterglow is expected to outshine its host galaxy, even more so at high redshifts, when the typical galaxies are less massive than their present-day counterparts (Barkana \& Loeb 2001). The broadband afterglow spectrum extends into the far-UV, and so the absorption features imprinted on it by the intervening intergalactic medium (IGM) can be used to infer the evolution of the neutral hydrogen fraction and the metal abundance of the IGM during the epoch of reioniza- tion. In difference from galaxies and quasars, which fade rapidly with increasing redshift because of the increase in their luminosity distance, GRB afterglows maintain an almost constant infrared flux with increasing redshift at a fixed time lag after the GRB trigger in the observer frame (Ciardi \& Loeb 2000). This follows from the cosmological time-stretching of the afterglow transient (which is intrinsically brighter at earlier times) and from a favorable $K$-correction in the afterglow spectrum.

The Swift satellite, ${ }^{1}$ planned for launch in 2003, is expected to localize roughly one GRB per day. Sorting out the subset of all GRBs that originate at high redshifts $(z \gtrsim 5)$ would be of particular interest. Observers may employ a simple strategy for this purpose. Photometric data from a small telescope should be used at first to identify those GRBs that possess a $\mathrm{Ly} \alpha$ trough at a wavelength of $0.73 \mu \mathrm{m}(1+z) / 6$ due to absorption by the IGM. Followup spectroscopy of those GRBs could then be done on a 10 $\mathrm{m}$ class telescope. In designing this observing strategy, it is important to forecast the fractions of all GRBs that originate at different redshifts. For example, it would be impractical to search for those very high redshifts that account for a fraction smaller than $10^{-3}$ of all GRBs, because barely a single one of them would be found by Swift over several years of operation.

In this paper, we use existing observational and theoretical work on the cosmic star formation history to predict the fractions of all GRBs that are expected to originate at different redshifts. In order to keep our results general, we make predictions about all GRBs without reference to the detection threshold or redshift horizon of any particular instrument. To ascertain, however, what the BATSE and Swift instruments are expected to detect, we in addition estimate the redshift distributions for these flux-limited surveys.

In $\S 2$, we calculate the collapsed fraction of baryons as a function of redshift, based on the Press-Schechter formalism, and infer the corresponding redshift distribution of

\footnotetext{
${ }^{1}$ See http://swift.gsfc.nasa.gov.
} 
GRBs. In $\S 3$, we use the inferred redshift distribution of GRBs to convert the observed distribution of GRB durations into the corresponding intrinsic distribution, under the simple assumption that its normalized form is redshiftindependent. Finally, we discuss the implications of our results in $\S 4$.

\section{STRUCTURE FORMATION MODEL}

\subsection{Star Formation History}

We adopt the popular view that the formation of cosmic structure has progressed hierarchically from small to large scales, according to a variant of the cold dark matter (CDM) model. Specifically, we assume a $\Lambda$ CDM model with density parameters in matter $\Omega_{m}=1-\Omega_{\Lambda}=0.3$ and in baryons $\Omega_{B}=0.045$, a Hubble constant of $h=$ $H_{0} / 100 \mathrm{~km} \mathrm{~s}^{-1} \mathrm{Mpc}^{-1}=0.7$, and a scale-invariant power spectrum of density fluctuations with an amplitude $\sigma_{8}=$ 0.9 on a scale of $8 h^{-1} \mathrm{Mpc}$.

Our star formation model closely follows that of Barkana \& Loeb (2000), and here we only briefly describe the key assumptions (see also Santos, Bromm, \& Kamionkowski 2002). The abundance and merger history of the CDM halos is described by the extended Press-Schechter formalism (Lacey \& Cole 1993). We assume that the IGM has a two-phase structure, consisting of a neutral and an ionized phase. The fraction of the cosmic volume filled with $\mathrm{H}$ II regions is given by

$$
f_{\text {ion }}=\left\{\begin{array}{lc}
\exp [-0.495(z-5.6)] & z>5.6 \\
1 & \text { otherwise } .
\end{array}\right.
$$

This assumed ionization history fits the semianalytical calculation of Barkana \& Loeb (2001) and is consistent with numerical simulations of reionization (Gnedin 2000, 2001; Razoumov et al. 2002) and the latest data on quasars in the redshift interval $5 \lesssim z \lesssim 6.3$ (Becker et al. 2001; Djorgovski et al. 2001a; Fan et al. 2002). At high redshifts, $z \gtrsim 20$, the universe is predominantly neutral. Once the first luminous objects form, an increasing fraction of the IGM becomes ionized. At $z_{\text {reion }} \approx 7$, the ionized phase in our model comprises a volume fraction of $\sim 50 \%$, and reionization of the IGM is complete by $z \approx 5.6$.

Within each phase of the IGM, stars are able to form in two different ways. The first mechanism pertains to primordial, metal-free, gas. Such gas undergoes star formation provided that it falls into a sufficiently deep CDM potential well, or equivalently, into a CDM halo more massive than a critical value. For the neutral medium, this minimum mass is set by the requirement for the gas to cool. Radiative cooling by molecular hydrogen $\left(\mathrm{H}_{2}\right)$ allows star formation in halos with a virial temperature $T_{\text {vir }} \gtrsim 300 \mathrm{~K}$, while atomic cooling dominates for halos with $T_{\mathrm{vir}} \gtrsim 10^{3.9} \mathrm{~K}$. The corresponding minimum circular velocities are $v_{c} \sim 2.5$ and $\sim 12$ $\mathrm{km} \mathrm{s}^{-1}$, respectively. Since $\mathrm{H}_{2}$ can be easily photodissociated by photons below the Lyman limit, its significance in the cosmic star formation history is unclear (see, e.g., Haiman, Abel, \& Rees 2000; Ricotti, Gnedin, \& Shull 2002, and references therein), and so we show results with and without $\mathrm{H}_{2}$ cooling. These two theoretical models are likely to provide conservative bounds for the true star formation history at $z \gtrsim 5$. The construction of more tightly constrained models has to await further advances in our understanding, both observational and theoretical, of star formation at the highest redshifts.

For the ionized medium, on the other hand, the minimum threshold mass is given by the Jeans mass, since the infall of gas and the subsequent formation of stars requires that the gravitational force of the collapsing CDM halo be greater than the opposing pressure force on the gas. After reionization, the IGM is photoheated to temperatures $\gtrsim 10^{4} \mathrm{~K}$, leading to a dramatic increase in the Jeans mass. We model the suppression of gas infall according to results from spherically symmetric collapse simulations (Thoul \& Weinberg 1996). Expressing the Jeans mass as the equivalent halo circular velocity, we assume complete suppression for halos with $v_{c} \lesssim 35 \mathrm{~km} \mathrm{~s}^{-1}$, no suppression for $v_{c} \gtrsim 93 \mathrm{~km} \mathrm{~s}^{-1}$, and a linear interpolation in between, so that $\sim 50 \%$ suppression occurs at $v_{c} \sim 55 \mathrm{~km} \mathrm{~s}^{-1}$.

Within our model, the second mechanism to form stars occurs in gas that has experienced a previous burst of star formation and is therefore already somewhat enriched with heavy elements. Such gas, residing in a halo of mass $M_{1}$, can undergo induced star formation triggered by a merger with a sufficiently massive companion halo of mass $M_{2}>0.5 M_{1}$. We finally assume that stars form with an efficiency of $\eta_{*} \sim 10 \%$, independent of redshift and regardless of whether the gas is primordial or preenriched. This efficiency yields roughly the correct fraction of $\Omega_{B}$ found in stars in the present-day universe.

Figure 1 shows the resulting star formation histories. Our theoretical models agree well with observational estimates of the cosmic star formation rate (SFR) at $z \lesssim 2$ (see, e.g., Blain et al. 1999). It is evident that there are two distinct epochs of cosmic star formation, one at $z \sim 3$ and a second one at $z \sim 8$ (or at even higher redshifts, if $\mathrm{H}_{2}$ cooling is effective). Again, we emphasize that the true history of the cosmic SFR is likely to lie between the two curves in Figure

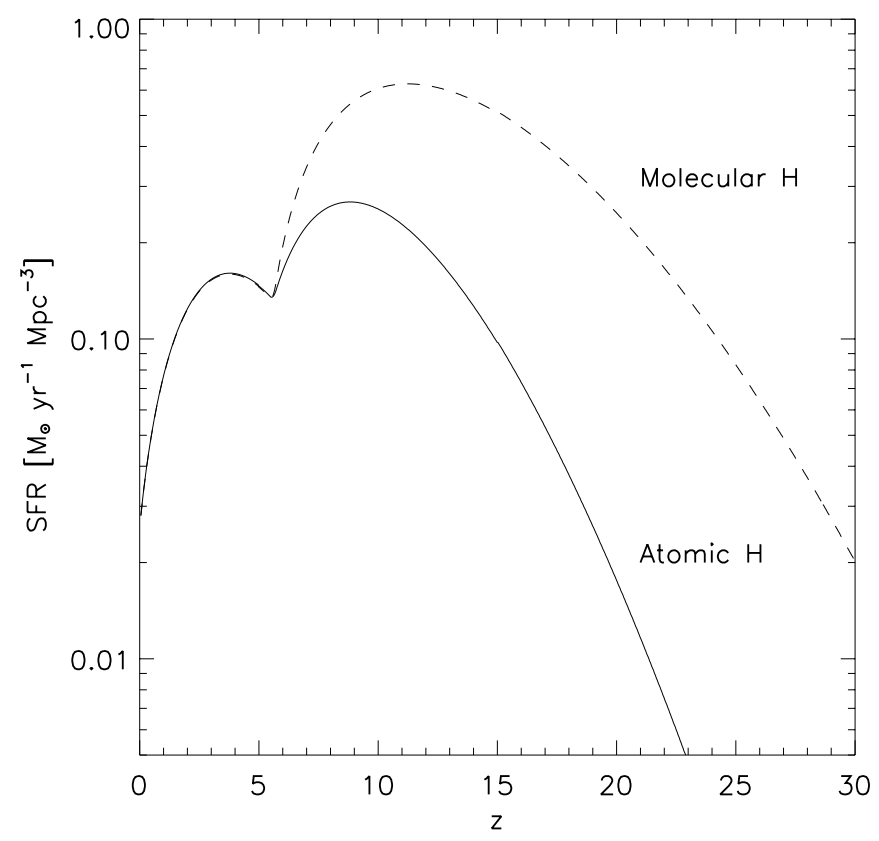

FIG. 1.-History of cosmic comoving star formation rate (SFR) in units of $M_{\odot} \mathrm{yr}^{-1} \mathrm{Mpc}^{-1}$, as a function of redshift. Solid line: Cooling due to atomic hydrogen only; dashed line: added cooling via molecular hydrogen. The star formation efficiency is assumed to be $\eta_{*}=10 \%$. 
1, depending on how complete the destruction of $\mathrm{H}_{2}$ as a function of redshift is.

In deriving the redshift distribution of GRBs in $\S 2.2$, we do not make any assumptions about the possible variation of the IMF for stars forming at different redshifts. Instead, we only assume that baryons are incorporated into stars, regardless of their specific properties, at the overall rate calculated in this section. Let us, however, briefly discuss the possible differences in star formation at high and low redshifts, based on recent theoretical work implying that star formation at high redshifts might have proceeded very differently from the present-day case, leading to stars with typical masses of $M_{*} \gtrsim 100 M_{\odot}$ (Bromm et al. 1999, 2002; Abel et al. 2000, 2002; Nakamura \& Umemura 2001). After the first stars had formed, the subsequent generation of stars formed out of gas that was already enriched with heavy elements. This enriched gas could have cooled more efficiently and thus could have been able to reach lower temperatures. Star formation, then, is expected to result in a less top-heavy IMF. As shown by Bromm et al. (2001), the transition from a top-heavy to the more standard (Salpeter) IMF occurs when the mass fraction in metals exceeds a critical value of $\sim 10^{-3} Z_{\odot}$. Gas with a metal abundance below this threshold is therefore still expected to form very massive stars. An IGM metal abundance of $\sim 10^{-3} Z_{\odot}$ approximately corresponds to the production of enough ionizing stellar photons to reionize the universe. Star formation at $z \gtrsim 7$ might consequently have been dominated by very massive stars, whereas at lower redshifts, stars form with an IMF close to the Salpeter form.

\subsection{Redshift Distribution of GRBs}

Under the assumption that the formation of GRBs follows closely the cosmic star formation history with no cosmologically significant time delay, we write for the number of GRB events per comoving volume per time: $\psi_{\mathrm{GRB}}(z)=\eta_{\mathrm{GRB}} \psi_{*}(z)$, where $\psi_{*}(z)$ is the stellar mass produced on average per comoving volume per time, as calculated in $\S 2.1$. The efficiency factor, $\eta_{\mathrm{GRB}}$, links the formation of stars to that of GRBs and is in principle a function of redshift, as well as of the properties of the underlying stellar population. Massive stars of Population I differ fundamentally from those of Population III; moreover, it is at present not well understood how a massive Population III star can give rise to a GRB (see Fryer, Woosley, \& Heger 2001; Schneider et al. 2002). Given the current state of uncertainty with regard to the central engine of GRBs (see, e.g., Piran 2000), we make the simplifying assumption that both populations of massive stars are connected to GRBs in a similar way and take $\eta_{\mathrm{GRB}}$ to be independent of redshift. While this simplifying assumption follows from the lack of better information, our analysis will provide a starting point for future improvements as soon as better observational constraints on high-redshift GRB and star formation become available.

If we now consider a time interval $\Delta t_{\mathrm{obs}}$ in the observer frame, the total number of GRBs, regardless of whether they are actually observed or missed, can be written as

$$
N(>z)=\int_{z}^{\infty} \psi_{\mathrm{GRB}}\left(z^{\prime}\right) \frac{\Delta t_{\mathrm{obs}}}{\left(1+z^{\prime}\right)} \frac{d V}{d z^{\prime}} d z^{\prime},
$$

where $d V / d z$ is the comoving volume element per unit red- shift, given by

$$
\frac{d V}{d z}=\frac{4 \pi c d_{L}^{2}}{1+z}\left|\frac{d t}{d z}\right| .
$$

The luminosity distance, $d_{L}$, to a source at redshift $z$ is

$$
d_{L}=c(1+z) \int_{0}^{z}\left(1+z^{\prime}\right)\left|\frac{d t}{d z^{\prime}}\right| d z^{\prime},
$$

with

$$
\left|\frac{d t}{d z}\right|^{-1}=(1+z) H_{0} \sqrt{\Omega_{m}(1+z)^{3}+\Omega_{\Lambda}},
$$

in a flat universe. The fraction of bursts that originate at a redshift of $z$ or higher, $f(>z)=N(>z) / N(>0)$, is independent of constant parameters, such as $\Delta t_{\mathrm{obs}}, \eta_{\mathrm{GRB}}$, or the beaming factor of the GRB emission. The integrand in equation (2) contains the differential comoving volume element, $d V / d z$, as is appropriate for the calculation of an event rate. Since GRB events are communicated via photons, we integrate over the redshift-dependent comoving volume element along our past light cone. We observe these events over a fixed time window, $\Delta t_{\mathrm{obs}}$, which corresponds to $\Delta t_{\mathrm{obs}} /(1+z)$ in the source frame. If, on the other hand, we were interested in determining the amount of stellar fossils that have accumulated over cosmic time in a local comoving volume (see below), we would have to simply integrate over cosmic time along our past worldline.

In Figure 2, we show $f(>z)$, together with the differential distribution $(d f / d z)$, for the two star formation histories of Figure 1. It is evident that a significant fraction of all bursts are predicted to occur at high redshifts, namely, $f(z \geq 5) \geq 50 \%$, and that a few percent of all bursts occur at redshifts as high as $z \sim 20$. Evaluating the mean redshift for

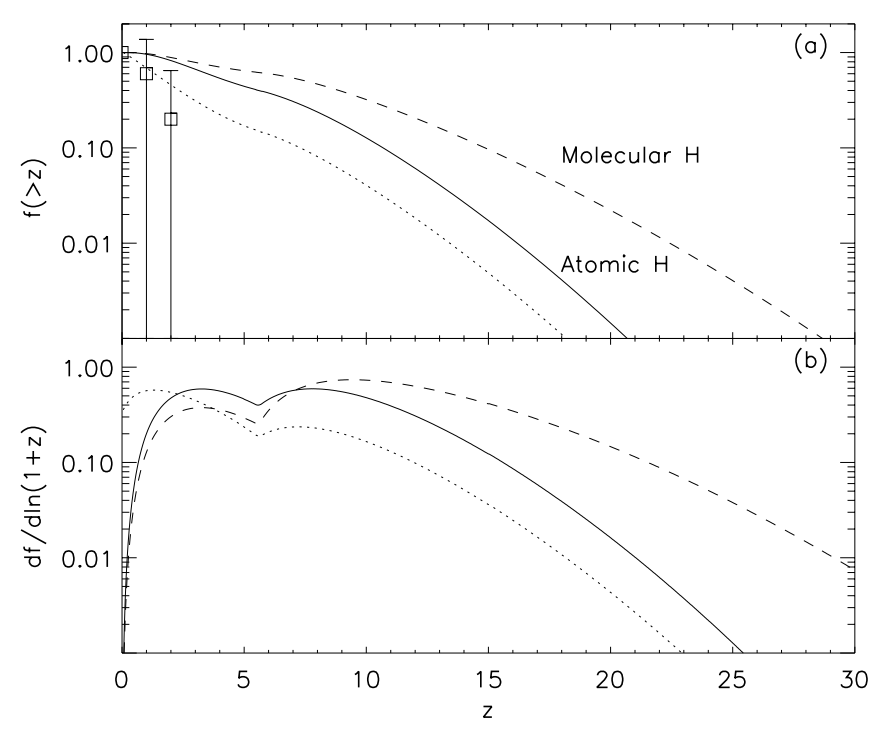

FIG. 2.-Redshift distribution of GRBs. (a) Fraction of bursts that originate at a redshift higher than $z$ vs. $z$. The curves correspond to the two star formation histories in Fig. 1. The data points reflect $\sim 20$ observed redshifts (from Ghisellini 2001). (b) Fraction of bursts per logarithmic interval of $(1+z)$ vs. $z$. The curves have the same meaning as in $(a)$. Dotted lines: Redshift distribution of the baryonic mass fraction in stars, calculated for the case of atomic hydrogen cooling. 
GRBs using the distributions of Figure 2, we find $\bar{z} \sim 5$ in the case of atomic cooling and $\bar{z} \sim 8$ for molecular cooling. Currently, only $\sim 20$ bursts have known redshifts (Kulkarni et al. 2000; Djorgovski et al. 2001c; Ghisellini 2001), and we include this small observed sample in Figure 2. The fact that the data points lie below our theoretical prediction could be due to small-number statistics, as well as to a redshiftdependent incompleteness bias.

We stress that equation (2) gives the fraction of transient events observed on the sky, and not the fraction of all baryons that have been incorporated into stars by a redshift $z$ relative to the same fraction today. This latter quantity is given by

$$
f_{*}(>z)=\frac{\int_{z}^{\infty} \psi_{*}\left(z^{\prime}\right)\left|d t / d z^{\prime}\right| d z^{\prime}}{\int_{0}^{\infty} \psi_{*}\left(z^{\prime}\right)\left|d t / d z^{\prime}\right| d z^{\prime}},
$$

and is shown in Figure 2 for the case of atomic cooling. As can be seen, the fraction of all stars that are formed at $z \gtrsim 5$ is $\sim 15 \%$. Collecting photons from our past light cone is therefore a more efficient way of probing the first stars than sorting through the fossil stellar record in the present-day universe.

It is important to emphasize that the analysis presented here pertains to all bursts, regardless of whether existing or previous instruments have actually been able to detect them. If the horizon of previous instruments was limited to $z \ll 5$, then our predictions provide important motivation for the construction of more sensitive instruments that would trigger on GRBs out to the highest redshifts. The fraction of all bursts detected by any given instrument depends on the instrument-specific flux sensitivity threshold and on the poorly determined luminosity function (LF) of GRBs (see, e.g., Schaefer, Deng, \& Band 2001; Schmidt 2001; Norris 2002).

It is nevertheless instructive to ascertain what existing or planned instruments like BATSE and Swift are expected to find. To this extent, we modify the GRB event rate to have

$$
\psi_{\mathrm{GRB}}(z)=\eta_{\mathrm{GRB}} \psi_{*}(z) \int_{L_{\mathrm{lim}}(z)}^{\infty} p(L) d L .
$$

Here, $p(L)$ is the GRB LF, with $L$ being the intrinsic photon luminosity (in units of photons $\mathrm{s}^{-1}$ ). If $f_{\text {lim }}$ denotes the sensitivity threshold of a given instrument (in photons $\mathrm{s}^{-1} \mathrm{~cm}^{-2}$ ), then the minimum luminosity is

$$
L_{\lim }(z)=4 \pi d_{L}^{2} f_{\lim } .
$$

This expression is derived with a spectral index of $\alpha=2$ for $L \propto \nu^{-\alpha}$ (Band et al. 1993). For definiteness, we assume a lognormal distribution function

$$
p(L)=\frac{e^{-\sigma^{2} / 2}}{\sqrt{2 \pi \sigma^{2}}} \exp \left\{-\frac{\left[\ln \left(L / L_{0}\right)\right]^{2}}{2 \sigma^{2}}\right\} \frac{1}{L_{0}},
$$

(see, e.g., Woods \& Loeb 1995), where $\sigma$ and $L_{0}$ are the width and the average luminosity, respectively. Recently, Sethi \& Bhargavi (2001) have shown that both the observed number count-flux relation and the existing afterglow redshift data are consistent with a lognormal LF for best-fit parameters (taking into account the effect of beaming) $\sigma=2$ and $L_{0}=2 \times 10^{56} \mathrm{~s}^{-1}$, and we adopt these values in the following analysis.

To determine the expected redshift distribution as observed by BATSE and Swift, we use equation (2), together with the GRB rate given in equation (7). The flux thresholds are $f_{\text {lim }}=0.2$ and 0.04 photons $\mathrm{s}^{-1} \mathrm{~cm}^{-2}$ for BATSE and Swift, respectively (Lamb \& Reichart 2000 and references therein). In Figure 3, we show the same quantities as in Figure 2, but now comparing the distributions for BATSE and Swift with our theoretical prediction for atomic line cooling. It can be seen that in the case of BATSE, a fraction $[f(z \geq 5) \geq 10 \%$ ] of all bursts originate at high redshifts, whereas the corresponding fraction for Swift is $f(z \geq 5) \gtrsim 25 \%$. We emphasize again that these numbers are uncertain because of the poorly known GRB LF. Figure 3 nicely demonstrates the asymptotic character of our theoretical prediction, pertaining to a future "ultimately sensitive" instrument. Indeed, using the LF above, we estimate that an instrument with a sensitivity of $\sim 50$ times better than Swift's would be able to detect the full theoretically possible sample of bursts from $z \gtrsim 5$.

The detectability of $z \gtrsim 5$ GRBs is also a crucial ingredient in estimating the fraction of all well-localized bursts that have no detectable optical afterglow, the so-called dark GRBs. Various authors have used the fraction of dark bursts in the currently observed sample of GRBs to constrain the amount of dust-obscured star formation (see, e.g., Djorgovski et al. 2001b). The resulting fractions of dark GRBs estimated for different redshifts depend on the currently unknown level of incompleteness in the observed sample. In the context of our model, we predict that all GRB afterglows originating at $z \gtrsim 6$ are optically dark. The intervening, partially neutral IGM would efficiently absorb the rest-frame UV afterglow that would otherwise have been redshifted into the optical band (see also Fruchter 1999; Piro et al. 2002). These bursts might give rise to the recently discovered class of X-ray-rich GRBs (see, e.g., Piro et al. 2002; see also Schneider et al. 2002) as a result of the redshifting of the source-frame $\gamma$ rays into the $\mathrm{X}$-ray band.

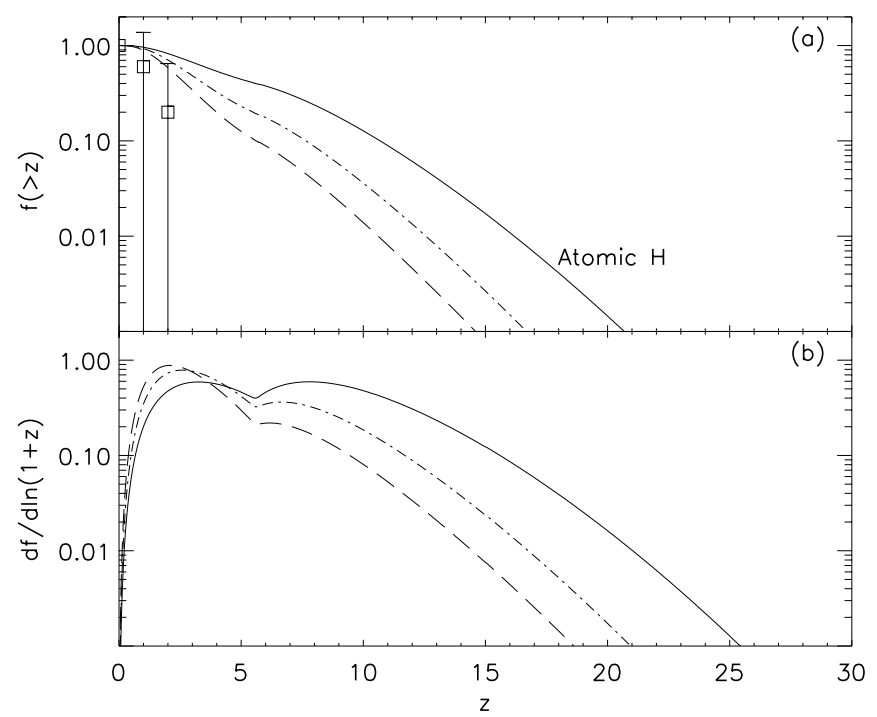

FIG. 3.- Redshift distribution of all GRBs as compared with that measured by flux-limited surveys. Conventions for the panels and symbols are the same as in Fig. 2. Solid lines: All GRBs for star formation through atomic line cooling. Dot-dashed lines: Expected distribution for Swift. Long-dashed lines: Expected distribution for BATSE. Note that the curves for the two flux-limited surveys are very uncertain because of the poorly determined GRB LF. 


\section{BURST DURATIONS}

The duration of a GRB reflects the characteristic timescale over which the central engine is active and is therefore a diagnostic of the GRB progenitor. The distribution of GRB durations has been determined by the BATSE instrument on board the Compton Gamma Ray Observatory (as summarized in Paciesas et al. 1999) and is observed to be bimodal, with a population of short bursts centered on $T_{\text {obs }} \sim 0.3 \mathrm{~s}$ and one of long bursts around $T_{\text {obs }} \sim 30 \mathrm{~s}$ (Kouveliotou et al. 1993). For the definition of the burst duration, $T_{\text {obs }}$, we use the interval of time over which a GRB contains from $5 \%$ to $95 \%$ of its total observed $\gamma$-ray counts, also denoted as $T_{90}$ in the literature. Since bursts originate over a broad range of redshifts, the question arises as to what the intrinsic distribution of durations is like. For simplicity, we assume in this section that BATSE was capable of sampling the full redshift distribution of GRBs shown in Figure 2. This provides us with the maximum level of distortion that cosmological time dilation could have had on the observed distribution of burst durations. The true intrinsic distribution of durations for the BATSE-triggered bursts should lie between the observed distribution and the intrinsic one calculated in this section.

The number of observed bursts in a given bin $i$, with an observed duration $T_{\mathrm{obs}, i}$ and a width $\Delta T_{\mathrm{obs}, i}$, can be written as

$$
\Delta N_{\mathrm{obs}, i}=N_{\mathrm{tot}} \Delta T_{\mathrm{obs}, i} \int_{0}^{\infty} \frac{d P}{d T}(T) \frac{1}{(1+z)} \frac{d f}{d z}(z) d z,
$$

where $N_{\text {tot }}=\sum_{i} \Delta N_{\text {obs, } i}$ is the total number of bursts in the sample. We assume that the intrinsic distribution, $d P / d T$, is independent of redshift and satisfies $\int_{0}^{\infty}(d P / d T) d T=1$ and $(d P / d T) \geq 0$. The intrinsic burst duration, $T$, is related to the observed one by the cosmological time dilation, $T=T_{\text {obs, } i} /(1+z)$, and $(d f / d z)$ is the GRB redshift distribution, as calculated in $\S 2.2$. We replace the integration in equation (10) by a summation covering the range of intrinsic durations, $T$, with the same number of bins, $N_{\text {bin }}=23$, as the observed histogram. The inversion problem is then uniquely defined. We carry out the deconvolution with the standard iterative Lucy method. This is a reliable technique, derived from Bayes's theorem, to solve a set of linear equations with additional constraints on the unknowns (Lucy 1974). The stability of the algorithm is improved by limiting the change in the unknowns in each iteration and by smoothing over adjacent bins. To this extent, we use equation (11b) of Baugh \& Efstathiou (1993), with parameter values of $\beta=0.8$ and $\epsilon=0.9$. We have verified that the solutions are not very sensitive to the choice of these parameters.

The result of this inversion is shown in Figure 4, where we compare the derived intrinsic distribution with the observed one, $d P / d T_{\text {obs }}\left(T_{\text {obs }, i}\right)=\Delta N_{\text {obs }, i} / N_{\text {tot }} \Delta T_{\text {obs }, i}$. It is evident that the intrinsic durations are systematically shifted to shorter values as a result of the cosmological time dilation. The bimodality is preserved, with peaks that are narrower than the observed ones (note that the horizontal scale is logarithmic). The two star formation histories discussed in $\S 2.1$ lead to similar intrinsic distributions. The shift to shorter durations, however, is more pronounced in the case of star formation via $\mathrm{H}_{2}$ cooling. The mean intrinsic durations characterizing the first, short-duration peak are $\sim 0.05 \mathrm{~s}$ for cooling due to atomic hydrogen and $\sim 0.03 \mathrm{~s}$ for $\mathrm{H}_{2}$ cooling.

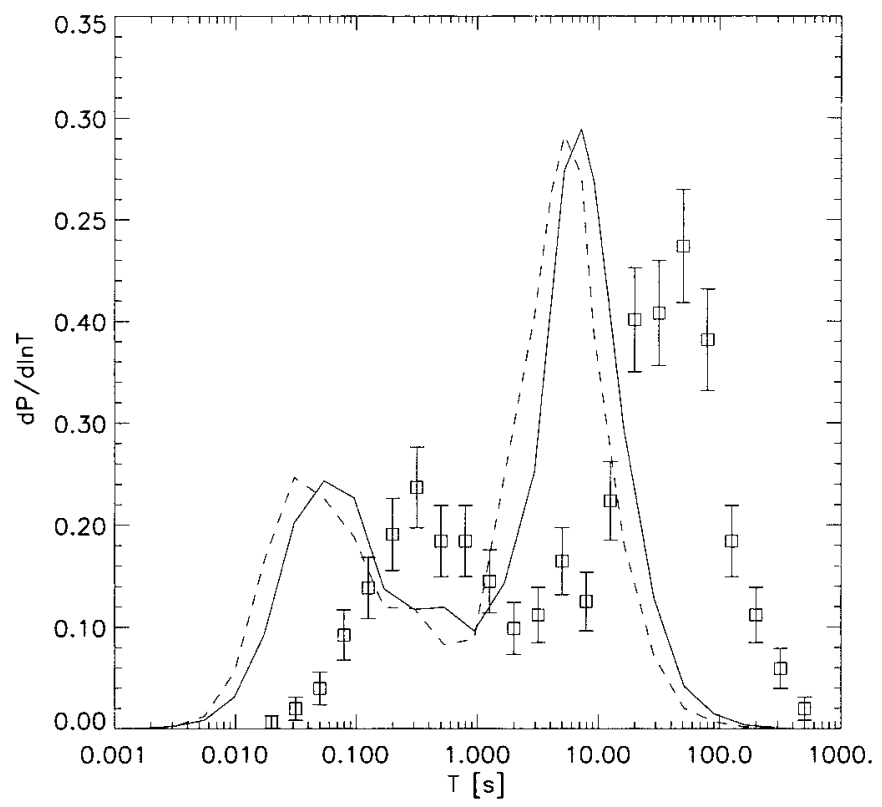

FIG. 4.-Maximal effect that cosmological time dilation can have on the observed distribution of GRB durations. Distributions are shown as probability per logarithmic duration interval vs. duration (in seconds). Solid line: Case of atomic hydrogen cooling. Dashed line: Case with molecular cooling included as well. The data points correspond to the observed distribution by BATSE (Paciesas et al. 1999). The intrinsic durations are systematically shifted to smaller values as a result of cosmological time dilation.

The corresponding numbers for the long-duration peak are $\sim 7$ and $\sim 5 \mathrm{~s}$, respectively. These differences in the mean durations are a direct consequence of the fact that GRBs originate, on average, at somewhat higher redshift if $\mathrm{H}_{2}$ cooling is effective. Note that, statistically, the longest duration bursts, with $T_{\text {obs }} \gtrsim 1000 \mathrm{~s}$, are expected to originate at high $z$, and this could be a successful selection strategy for observations targeting high-redshift GRBs. The shift to longer durations due to the cosmological time dilation could in part be compensated for by the following subtle selection effect, which we ignore in this paper. Sources at high $z$ will on average have lower fluxes, and observations with a given sensitivity threshold will therefore only detect the brightest portion of the total emission, thus systematically underestimating the true duration of the burst.

Based on observations of GRB afterglows with known redshifts, Frail et al. (2001) have recently presented evidence for a standard amount of energy release in GRBs, $E \sim 10^{51}$ ergs. Making the simplest assumption of a constant energy for all long-duration bursts (which are the only ones with measured redshifts so far), one can easily derive the LF from the intrinsic distribution of burst durations. The luminosity of a burst is then simply $\approx E / T$, and the resulting $L F$ is obtained by inverting the horizontal axis in Figure 4 and changing $T$ to $E / T$. The long-duration bursts would then narrowly cluster around a luminosity of $\sim 10^{50} \mathrm{ergs} \mathrm{s}^{-1}$.

\section{DISCUSSION}

We have derived the redshift distribution of GRBs out to $z \gtrsim 20$, under the assumption that the GRB event rate traces the cosmic SFR. We find that $\gtrsim 50 \%$ of all GRBs on the sky originate at a redshift of 5 or higher. On the other hand, the fraction of baryons that has been incorporated into stars by 
$z \sim 5$ is much smaller, comprising only $\sim 15 \%$ of the stellar mass formed by today. The difference between the two fractions follows from the different cosmological factors in the redshift integrations for the statistics of transient events on the sky, as compared with the census of fossil objects in the local universe. The favorable statistical bias toward highredshift events on the sky is expected to apply also to Type II supernova explosions, which are related to the formation of massive stars in a similar way as GRBs. Despite their dimming with increasing redshift, high-redshift supernovae will be detectable with sufficiently sensitive telescopes, such as the Next Generation Space Telescope (NGST; MiraldaEscudé \& Rees 1997; Woods \& Loeb 1998). ${ }^{2}$ In fact, our calculation implies that without any additional bias (such as redshift-dependent dust extinction), approximately half of all Type II supernovae detected by NGST will originate at $z \gtrsim 5$. Deep observations of high-redshift GRBs and supernovae offer an ideal window into the earliest epoch of cosmic structure formation. The lengthening of the duration of these transients by a factor $(1+z)$ makes it easier for observers to monitor their light curves.

Different instruments may find GRBs up to different redshifts, depending on their detection sensitivity and the

\footnotetext{
${ }^{2}$ See http://ngst.gsfc.nasa.gov.
}

highly uncertain GRB LF (Schaefer et al. 2001; Schmidt 2001; Norris 2002). A trigger-unbiased way to infer the redshift evolution of the GRB event rate is to compare the number counts of GRBs with the same absolute (intrinsic) luminosity in different redshift bins. If future observations of this type were to determine a mean redshift for the GRB distribution significantly lower than the one predicted in this paper, then this would indicate either that GRB formation at high $z$ is substantially suppressed or that GRBs originate from the coalescence of binaries with a time delay of a few gigayears between the formation of a massive star and the GRB event.

Recent observations indicate that a large fraction, $\sim 50 \%$, of all well-localized GRBs have no associated optical afterglow and are classified as (optically) dark GRBs (see, e.g., Piro et al. 2002). According to our model, a substantial fraction of these dark bursts could originate at $z \gtrsim 6$. The intervening, partially neutral IGM would efficiently absorb the rest-frame UV afterglow that would otherwise have been redshifted into the optical band.

We thank Rennan Barkana, Jeremy Heyl, Jonathan Mackey, Bohdan Paczyński, and Martin Rees for helpful discussions. This work was supported in part by NASA grants NAG5-7039 and NAG5-7768, and by NSF grants AST 99-00877 and AST 00-71019.
Abel, T., Bryan, G. L., \& Norman, M. L. 2000, ApJ, 540, 39 2002, Science, 295, 93

Band, D., et al. 1993, ApJ, 413, 281

Barkana, R., \& Loeb, A. 2000, ApJ, 539, 20

2001, Phys. Rep. 349, 125

Baugh, C. M.. \& Efstathiou, G. 1993, MNRAS, 265, 145

Becker, R. H., et al. 2001, AJ, 122, 2850

Blain, A. W., Jameson, A., Smail, I., Longair, M. S., Kneib, J.-P., \& Ivison, R. J. 1999, MNRAS, 309, 715

Blain, A. W., \& Natarajan, P. 2000, MNRAS, 312, L35

Bloom, J. S., et al. 1999, Nature, 401, 453

Bromm, V., Coppi, P. S., \& Larson, R. B. 1999, ApJ, 527, L5

. 2002, ApJ, 564, 23

Bromm, V., Ferrara, A., Coppi, P. S., \& Larson, R. B. 2001, MNRAS, 328, 969

Ciardi, B., \& Loeb, A. 2000, ApJ, 540, 687

Djorgovski, S. G., Castro, S., Stern, D., \& Mahabal, A. A. 2001a, ApJ, 560, L5

Djorgovski, S. G., Frail, D. A., Kulkarni, S. R., Bloom, J. S., Odewahn, S. C., \& Diercks, A. 2001b, ApJ, 562, 654

Djorgovski, S. G., et al. 2001c, in Gamma-Ray Bursts in the Afterglow Era, ed. E. Costa, F. Frontera, \& J. Hjorth (Berlin: Springer), 218

Eichler, D., Livio, M., Piran, T., \& Schramm, D. N. 1989, Nature, 340, 126

Fan, X., Narayanan, V. K., Strauss, M. A., White, R. L., Becker, R. H.

Pentericci, L \& Rix, H.-W, 2002, AJ, 123, 1247

Frail, D. A., et al. 2001, ApJ, 562, L55

Fruchter, A. S. 1999, ApJ, 512, L1

Fryer, C. L., Woosley, S. E., \& Heger, A. 2001, ApJ, 550, 372

Ghisellini, G. 2001, preprint (astro-ph/0111584)

Gnedin, N. Y. 2000, ApJ, 535, 530

2001, MNRAS, submitted (astro-ph/0110290)

Haiman, Z., Abel, T., \& Rees, M. J. 2000, ApJ, 534, 11
REFERENCES

Janka, H.-T., Eberl, T., Ruffert, M., \& Fryer, C. L. 1999, ApJ, 527, L39

Kouveliotou, C., Meegan, C. A., Fishman, G. J., Bhat, N. P., Brigos, M. S. Koshut, T. M., Paciesas, W. S., \& Pendleton, G. N. 1993, ApJ, 413, L101 Kulkarni, S. R., et al. 2000, Proc. SPIE, 4005, 9

Lacey, C., \& Cole, S. 1993, MNRAS, 262, 627

Lamb, D. Q., \& Reichart, D. E. 2000, ApJ, 536, 1

Lucy, L. B. 1974, AJ, 79, 745

MacFadyen, A. I., Woosley, S. E., \& Heger, A. 2001, ApJ, 550, 410

Miralda-Escudé, J., \& Rees, M. J. 1997, ApJ, 478, L57

Nakamura, F. \& Umemura, M. 2001, ApJ, 548, 19

Norris, J. P. 2002, ApJ, submitted (astro-ph/0201503)

Paciesas, W. S., et al. 1999, ApJS, 122, 465

Piran, T. 2000, Phys. Rep., 333, 529

Piro, L., et al. 2002, ApJ, submitted (astro-ph/0201282)

Porciani, C., \& Madau, P. 2001, ApJ, 548, 522

Razoumov, A. O., Norman, M. L., Abel, T., \& Scott, D. 2002, ApJ, 572, 695

Reichart, D. E. 2001, ApJ, 554, 643

Ricotti, M., Gnedin, N. Y., \& Shull, J. M. 2002, ApJ, 575, 33

Santos, M. R., Bromm, V., \& Kamionkowski, M. 2002, MNRAS, submitted (astro-ph/0111467)

Schaefer, B. E., Deng, M., \& Band, D. L. 2001, ApJ, 563, L123

Schmidt, M. 2001, ApJ, 559, L79

Schneider, R., Ferrara, A., Natarajan, P., \& Omukai, K. 2002, ApJ, 571, 30

Sethi, S. \& Bhargavi, S. G. 2001, A\&A, 376, 10

Thoul, A. A., \& Weinberg, D. H. 1996, ApJ, 465, 608

Totani, T. 1997, ApJ, 486, L71

. 1999, ApJ, 511, 41

Wijers, R. A. M. J., Bloom, J. S., Bagla, J. S., \& Natarajan, P. 1998, MNRAS, 294, L13

Woods, E., \& Loeb, A. 1995, ApJ, 453, 583

. 1998, ApJ, 508, 760 